\title{
Veno-arterial extracorporeal membrane oxygenation may be useful in critically ill COVID-19 patients with suspected cardiac injury
}

\author{
Eunyoung Heo ${ }^{1}$, Se Jin $\mathrm{Oh}^{2}$, Hyun Woo Lee ${ }^{1}$, Jung-Kyu Lee ${ }^{1}$, Deog Kyeom Kim ${ }^{1}$, Sang-Won Park ${ }^{1}$ \\ Departments of ${ }^{1}$ Internal Medicine and ${ }^{2}$ Thoracic and Cardiovascular Surgery, Seoul Metropolitan Government-Seoul National University Boramae Medical \\ Center, Seoul, Korea
}

\section{Dear Editor:}

Recent studies reported that $12 \%-19.7 \%$ of patients with coronavirus disease-19 (COVID-19) exhibit cardiac injuries manifesting as an ejection fraction decline and troponin I elevation $[1,2]$. Cardiac injury is associated with higher mortality, the need for ICU care, and severe COVID-19 [3]. Recently, a 63-year-old man presented to Seoul Metropolitan GovernmentSeoul National University Boramae Medical Center with severe respiratory manifestations and fulminant myocarditis with an enlarged left ventricle and depressed left ventricular function (ejection fraction 32\%). He was placed on extracorporeal membrane oxygenation (ECMO). After treatment with intravenous immunoglobulin, steroids, and antiviral therapy, heart function recovered within 2 weeks [4].

We report a severe COVID-19 case of acute respiratory distress syndrome (ARDS) with suspected cardiac injury. Our findings suggest that veno-arterial (VA)-ECMO may be more beneficial than veno-venous (VV)-ECMO in ARDS patients with suspected cardiac injury.

A 61-year-old man with confirmed severe acute respiratory syndrome coronavirus 2 (SARSCoV-2) infection was admitted to the isolation ward on February 25, 2020. His comorbidities were hypertension, dyslipidemia, and atrial fibrillation. On admission, he had febrile sense, cough, and sputum. On hospital day 3, 5 days from initial symptom presentation, a high fever up to $38.9^{\circ} \mathrm{C}$ developed. This fever persisted, and on hospital day 7 , he felt a mild degree of shortness of breath on exertion, and room air oxygen saturation dropped from $95 \%$ to $92 \%$. Oxygen was immediately supplied via a nasal cannula. The next day, he needed high-flow nasal cannula oxygen therapy ( $70 \%$ concentration, flow $35 \mathrm{~L} / \mathrm{min}$ ) due to progressive hypoxemia. After 48 hours of initial oxygen therapy via nasal cannula, we decided to start mechanical ventilation. Despite mechanical ventilation support, $\mathrm{PaO}_{2} / \mathrm{FiO}_{2}$ ratio decreased from 81.62 to 70 over 2 hours. After starting mechanical ventilation, hemodynamic indicators worsened, and he required vasopressor support. His blood pressure was 83/55 mm Hg under $8 \mu \mathrm{g} / \mathrm{kg} /$ min norepinephrine administration. Although sedatives and muscle relaxants administered at the same time with mechanical ventilation might have affected his blood pressure, the patient's heart appeared enlarged compared with the initial chest X-ray (Figure 1). Given the rapid deterioration of hypoxemia and hemodynamic instability, we could not perform echocardiography to evaluate heart function before ECMO insertion. However, we suspected cardiac dysfunction in this patient due to progressive cardiomegaly and hypotension. Therefore,

\section{Letter to the Editor}

Received: May 14, 2020

Revised: May 27, 2020

Accepted: May 28, 2020

Corresponding author Sang-Won Park

Department of Internal Medicine, Seoul Metropolitan GovernmentSeoul National University Boramae Medical Center, Seoul National University College of Medicine, 20 Boramae-ro 5-gil, Dongjak-gu, Seoul 07061, Korea

Tel: +82-2-870-2114

Fax: +82-2-870-3863

E-mail: hswon1@snu.ac.kr

Copyright (@) 2020 The Korean Society of Critical Care Medicine

This is an Open Access article distributed under the terms of Creative Attributions Non-Commercial License (https:// creativecommons.org/li-censes/by-nc/4.0/) which permits unrestricted noncommercial use, distribution, and reproduction in any medium, provided the original work is properly cited. 

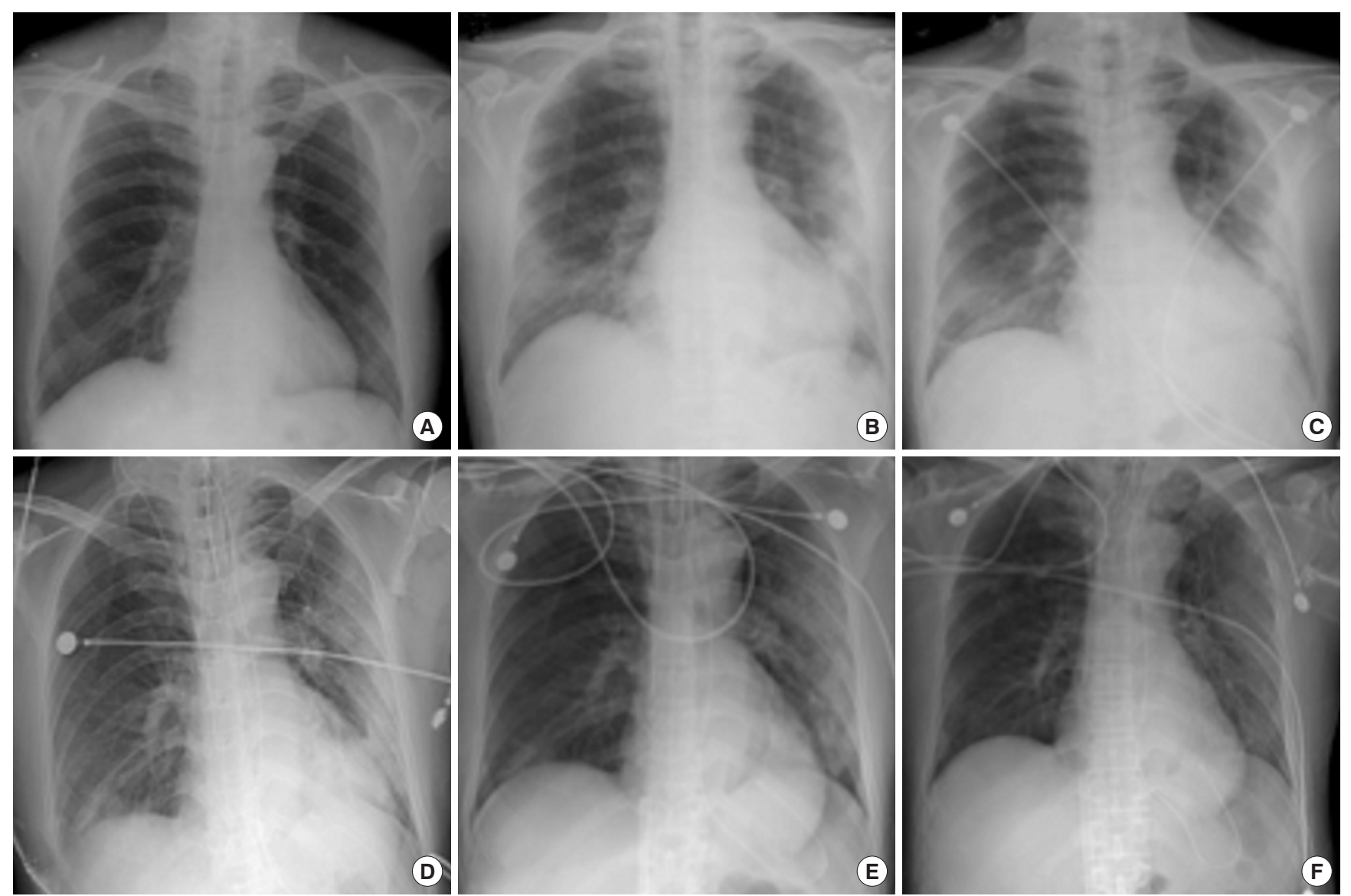

Figure 1. Change in cardiothoracic ratios. The cardiothoracic ratios decreased after VA-ECMO application. (A) At admission. (B) On highflow nasal cannula (hospital day [HD] 8). (C) Just before intubation (HD 9). (D) Ten hours on VA-ECMO (HD 10). (E) Three days on VA-ECMO (HD 12). (F) Five days on VA-ECMO (just before ECMO weaning; HD 14). VA-ECMO: veno-arterial extracorporeal membrane oxygenation.

VA-ECMO was inserted to support both heart and lung function. Shortly after VA-ECMO was applied, portable echocardiography was performed to assess the patient's heart function. Cardiac systolic function was severely depressed with a visually estimated ejection fraction of $20 \%-25 \%$. Markers of myocardial injury including troponin I and n-terminal fragment of pro-brain natriuretic peptide were elevated up to 37.4 $\mathrm{pg} / \mathrm{ml}(0-19.8 \mathrm{pg} / \mathrm{ml})$ and $615.5 \mathrm{pg} / \mathrm{ml}(0-194 \mathrm{pg} / \mathrm{ml})$, respectively. With VA-ECMO support, cardiac function rapidly improved. When we performed follow-up portable echocardiography on the following day, ejection fraction was $40 \%-45 \%$. After 5 days of use, we were able to wean the patient off VAECMO, and subsequently, 2 days after ECMO weaning, we were able to successfully wean him off the mechanical ventilator. He received an antiviral agent (lopinavir/ritonavir 400 $\mathrm{mg} / 100 \mathrm{mg}$ ) with empirical antibiotics for 20 days. He was finally discharged on hospital day 27 .

Given the rapid progression of both hypoxemia and cardiomegaly in this severe COVID-19 patient, we opted for VA-EC-
MO instead of VV-ECMO, suspecting that cardiac injury occurred concomitantly. The patient was able to be weaned off mechanical ventilator and ECMO within a week. Therefore, in severe COVID-19 cases complicated by ARDS and cardiac injury, VA-ECMO may be beneficial.

\section{CONFLICT OF INTEREST}

No potential conflict of interest relevant to this article was reported

\section{ORCID}

Eunyoung Heo Se Jin Oh

Hyun Woo Lee Jung-Kyu Lee Deog Kyeom Kim Sang-Won Park https://orcid.org/0000-0003-3803-4903 https://orcid.org/0000-0002-1516-5313 https://orcid.org/0000-0003-4379-0260 https://orcid.org/0000-0001-5060-7255 https://orcid.org/0000-0001-9379-8098 https://orcid.org/0000-0002-0550-1897 


\section{AUTHOR CONTRIBUTIONS}

Conceptualization: EH, SWP. Data curation: EH, SJO, HWL, JKL. Formal analysis: EH, SJO, HWL, JKL. Methodology: EH, SJO, HWL, JKL, DKK. Project administration: DKK, SWP. Visualization: EH. Writing-original draft: EH. Writing-review \& editing: all authors.

\section{REFERENCES}

1. Huang C, Wang Y, Li X, Ren L, Zhao J, Hu Y, et al. Clinical features of patients infected with 2019 novel coronavirus in $\mathrm{Wu}$ han, China. Lancet 2020;395:497-506.
2. Shi S, Qin M, Shen B, Cai Y, Liu T, Yang F, et al. Association of cardiac injury with mortality in hospitalized patients with COVID-19 in Wuhan, China. JAMA Cardiol 2020;5:802-10.

3. Santoso A, Pranata R, Wibowo A, Al-Farabi MJ, Huang I, Antariksa B. Cardiac injury is associated with mortality and critically ill pneumonia in COVID-19: a meta-analysis. Am J Emerg Med 2020 April 19 [Epub]. https://doi.org/10.1016/j.ajem.2020. 04.052 .

4. Zeng JH, Liu YX, Yuan J, Wang FX, Wu WB, Li JX, et al. First case of COVID-19 complicated with fulminant myocarditis: a case report and insights. Infection 2020 April 10 [Epub]. http: //doi.org/10.1007/s15010-020-01424-5. 